\title{
Un avenir pluriel et hybride
}

Introduction

A plural and hybrid future

Un futuro múltiple e híbrido

\section{Alain Bouvier}

\section{(2) OpenEdition}

\section{Journals}

Édition électronique

URL : https://journals.openedition.org/ries/710

DOI : $10.4000 /$ ries. 710

ISSN : 2261-4265

\section{Éditeur}

France Education international

\section{Édition imprimée}

Date de publication : 1 décembre 2009

Pagination : 25-30

ISBN : 978-2-8542-0577-0

ISSN : $1254-4590$

Référence électronique

Alain Bouvier, «Un avenir pluriel et hybride », Revue internationale d'éducation de Sèvres [En ligne], 52 | décembre 2009, mis en ligne le 30 juin 2011, consulté le 26 avril 2022. URL : http:// journals.openedition.org/ries/710; DOI : https://doi.org/10.4000/ries.710 


\title{
Un seul monde, une seule école?
}

\section{Introduction}

\section{Un avenir pluriel et hybride}

\begin{abstract}
Alain Bouvier
Le deuxième colloque international organisé, en 2009, par le Centre international d'études pédagogiques (CIEP) et la Revue internationale d'éducation de Sèvres fournit le corps de ce dossier consacré aux rapports entre "modèles scolaires » et «mondialisation ». C'est pour moi l'occasion de remercier le CIEP pour l'appui précieux apporté à l'organisation de ce colloque, et le comité d'organisation pour la conception scientifique de l'ensemble, notamment le choix des intervenants et des personnes ressources.

Pourquoi interroger ainsi les modèles scolaires ? Le terme même de « modèle » est-il approprié ? Faut-il, comme certains sociologues, Guy Vincent notamment ${ }^{1}$, lui préférer l'expression «forme scolaire " ?

Le modèle scolaire français et son principe d'équité, héritier des Lumières, a inspiré de nombreux pays. Tout au long des XIX et $\mathrm{XX}^{\mathrm{e}}$ siècles, l'École s'est construite d'une façon proche dans la majorité des territoires économiquement développés, tout en servant de référence un peu mythique aux pays en voie de développement. Ceux-ci étaient incités à agir en ce sens par les organismes internationaux (Banque mondiale, UNESCO, OCDE, etc.). Quelque soit le point de départ historique et le niveau de l'économie locale, il semblait admis que tous les États allaient, en gros, dans la même direction et que seule différait la rapidité d'évolution des systèmes éducatifs nationaux.
\end{abstract}

\section{L’ÉCOLE DU VILLAGE-PLANÈTE}

Cette hypothèse générale méritait d'être mise en question : aujourd'hui, malgré la multiplication des comparaisons internationales, des enquêtes et la publication de "standards", aucun élément ne prouve que l'École se dirige vers un unique modèle mondial. Au contraire, l'observation des réalités scolaires sur le «village planète» (selon les termes de Jacques Delors) ${ }^{2}$, semble montrer que, progressivement, émergent des systèmes différents et juxtaposés.

1. L'éducation prisonnière de la forme scolaire, PUL, 1994, Lyon.

2. Allocution de M. Jacques Delors, Président de la Commission internationale sur l'éducation pour le XXIe siècle à la vingt-huitième session de la Conférence générale de l'UNESCO, 3 novembre 1995. http://unesdoc.unesco.org/ images/0010/001018/101826fo.pdf/. 
Cet état de fait résulte de différences économiques et historiques, mais pas seulement. Les systèmes éducatifs n'ont pas comme intention première de converger vers un type d'École créé par quelques experts internationaux, ou vers un modèle qui serait directement importé d'un pays pris comme unique point de repère. Les territoires ne s'inscrivent pas dans une même logique éducative. Notamment, ils ne portent par les mêmes valeurs, ils ne promeuvent pas, pour l'École, le même rapport au système social, à l'économie et à l'emploi, à la religion, à la démocratie, à la citoyenneté, à l'égalité des sexes, au rôle des parents, des communautés, au traitement des minorités, etc. Leurs dissemblances résultent de l'histoire, de l'économie, de la géographie, des usages distincts des diverses technologies éducatives, mais aussi de changements sociétaux, de coutumes, de la variété des pratiques sociales, de leurs évolutions divergentes, de différentes traditions culturelles, philosophiques, religieuses et sans doute encore d'autres facteurs.

Certes, on constate qu'un peu partout, ou presque, s'emploient les mêmes mots ; mais ont-ils le même sens ? Au-delà de la question que cela pose, tout au long de la mise au point de ce dossier, il a fallu à Marie-José Sanselme et aux membres du comité de rédaction exercer une fine vigilance et assurer de nombreux allers-retours avec les auteurs. Les termes employés ne sont pas anodins et les traductions les déforment inévitablement. Par exemple, comme le note Tristan Lecoq, quand faut-il parler de "mondialisation » ou évoquer la " globalisation"? La vigilance linguistique s'imposait d'autant plus que les articles empruntent à la philosophie, à l'histoire, à la sociologie, à l'économie, aux sciences de l'éducation, à la géographie, à l'écologie... J'observe qu'au-delà des termes, tous les auteurs, chacun à sa façon et plus ou moins explicitement, parlent de valeurs, ce qui n'est pas le moindre pour des questions d'éducation.

\section{UN PATCHWORK MONDIAL}

Dans ce « monde fragmenté » (Thierry Breton), où " l'École a cessé d'être une solution pour devenir un problème » (Rui Canario), que croit-on discerner ?

Dans les lieux à grand potentiel économique et à forte densité de ressources scientifiques et culturelles, comme en Asie du Sud-Est, l'élitisme est affiché. Les élèves sont prévenus et les familles encouragent (financièrement notamment) cette orientation où, en complément de "l'école formelle », on observe que "l'École hors l'École » joue un rôle de plus en plus déterminant.

Dans les pays à très grande étendue géographique et à faible densité humaine, comme certaines provinces des États d'Amérique du Sud ou d'Afrique, le rapport à l'éducation est spécifique. Non seulement la "massification » n'est pas en place, mais elle n'est plus affichée comme objectif à moyen terme. Que veulent aujourd'hui les nations concernées?

On dit qu'il y aurait en Europe du Nord un type d'écoles performantes, reflet de leur nation, du contrat social et de la culture de ces pays. Qu'en est-il ? 
Certains États misent résolument sur des usages intensifs des nouvelles technologies et créent une "e-école ", comme en Corée du Sud. Ces technologies contribuent-elles aux mêmes apprentissages, sociaux notamment ? L'avenir de l'École est-il dans cette direction?

Une « quatrième voie » (Andy Hargreaves et Denis Shirley ${ }^{3}$ ) serait en développement : les "écoles de communautés », depuis les petites communautés familiales jusqu'à celles attachées à des courants de pensée, des idéologies, des religions, voire des sectes. S'agit-il d'une «tendance lourde » mondiale?

Y a-t-il opposition entre d'un côté une École ancrée sur des formes historiques, sanctuarisées, et d'un autre côté une "École sociétale », reflet direct du système social de proximité, incluant "l'école informelle ", comme cela semble le cas ici ou là ? Les mêmes finalités sont-elles visées ?

Dans ce patchwork mondial à peine esquissé ici, quelles convergences et quelles divergences discerne-t-on ? Quels sont les modèles en développement? S'inscrivent-ils dans les désormais classiques scénarios que l'OCDE a proposés en 2001 ? Repère-t-on les limites de la «massification " de l'École ? Vers quoi se dirige-t-on ? Est-il audacieux de considérer que les nouvelles formes scolaires naîtront dans les petits pays (Singapour, Écosse...), véritables laboratoires, porteurs d'audace et d'avenir ? Peut-on préciser à quoi ressemblera l'ensemble mondial qui se dessine à peine ? Avec le recul critique nécessaire, ce dossier tente de s'y employer.

\section{L’ÉCOLE TRAVERSÉE DE TENSIONS INTELLECTUELLES}

Ce numéro invite les lecteurs à se placer dans des « tensions » intellectuelles sources de débats, entre les vues économiques et écologiques, entre les approches historiques et sociologiques, entre les perspectives philosophiques, politiques, idéologiques (peut-on évoquer l'École sans références idéologiques?) et technologiques (voire technocratiques), administratives (la question de la gouvernance) et pédagogiques (reposant sur la mesure des acquis des élèves). Les textes qui le composent n'ont pas tous le même statut.

Thierry Breton, ancien ministre et professeur à Harvard, ouvre ce dossier par une lecture économique des mécanismes de mondialisation. Ce véritable cours de macroéconomie insiste sur le "monde fragmenté " actuel, mais aussi sur le rôle majeur des "communautés ». À sa pensée répond, en contrepoint, l'article philosophique, idéologique et historique du professeur Pavel Zgaga, ancien ministre de l'éducation en Slovénie. Entre ce qu'il nomme le "cosmopolitisme » et la "globalisation ", il note, dans son bilan historique du projet kantien, comme plus loin Roger Dale, la présence d'une étape nationale et un rôle particulier de l'Europe. Professeur à l'université de Bristol, Roger Dale

3. The Fourth Way. The Inspiring Future for Educational change, 2009, Cornwell press. 
change la perspective. Il s'exprime en lecteur attentif et critique des évolutions planétaires contemporaines. Il nous invite à prendre de la distance par rapport aux questions posées (pourquoi nous interrogeons-nous en ces termes ?) et à distinguer les modèles nationaux, européens et mondiaux.

Ces tensions intellectuelles culminent à la fin du dossier avec l'article de Riccardo Petrella, professeur émérite à l'université Louvain-la-Neuve, expert international sur les questions d'écologie. Il décrit l'éducation comme un bien commun, à l'image de l'eau et de la justice.

De l'économie au développement durable, tout ce qui borde l'école et ses évolutions est donc convoqué dans ce dossier dont le cœur est bien l'élève et ses apprentissages. Il interroge les pratiques pédagogiques des enseignants, l'organisation des systèmes éducatifs et surtout les valeurs qui les sous-tendent.

\section{Cinq aXes de Questionnement}

La description d'une grande variété de situations exige de disposer de témoignages précis, sous la forme de recherches ou d'études conduites en des lieux multiples, et de préférence présentés par celles et ceux qui les conduisent. Encore faut-il pouvoir les questionner, ne pas se contenter d'impressions premières parfois superficielles, ne pas seulement les ajouter platement les uns aux autres. Les cinq articles centraux du dossier mettent à profit des études de cas d'une trentaine de pays de tous les continents, du Nord et du Sud, de cultures et d'histoires diverses, de pays en voie de développement comme de pays en pointe sur l'usage scolaire des nouvelles technologies. Ils permettent de dégager une première série de constats. Chaque animateur d'atelier propose, dans un article, sa lecture de la problématique générale " Un seul monde, une seule école ? ", à travers un éclairage particulier (les héritages, les inégalités, les technologies...) et en s'appuyant sur les éléments les plus remarquables présentés dans son atelier ${ }^{4}$.

Autour de l'École et de ses héritages, des résistances aux changements, Françoise Martin van der Haegen offre une lecture du rôle de l'État-nation faisant référence à l'Histoire, illustrée à travers les cas du Japon, de la Hongrie, du Cameroun, des États-Unis, du Portugal et de la France. Les systèmes éducatifs de ces pays ont connu de nettes évolutions ces dernières décennies, mais de façon notablement distincte. Elle se risque à discerner les héritages et le sens des évolutions.

L'importance grandissante du rôle des standards internationaux pour l'évaluation des élèves et de l'École nécessitait de leur accorder une attention spécifique. L'article de Jean-Marie De Ketele s'appuie sur des éclairages venus de Suisse, de France et du Brésil, ainsi que sur des considérations à l'échelle de deux continents : l'Afrique et l'Europe. Il répond à la question de savoir s'il faut

4. Les contributions présentées le 13 mars 2009 lors des six ateliers sont consultables en ligne sur : http://www.ciep. $\mathrm{fr} /$ ries/index.php. 
plutôt rechercher des standards internationaux désincarnés (voire technocratiques) ou au contraire travailler avec des indicateurs spécifiques, contextualisés mais difficilement comparables.

Les évolutions des systèmes éducatifs vont-elles contribuer à diminuer ou à accroître les inégalités ? Nous dirigeons-nous vers plus d'équité ou, au contraire, sommes-nous face à une « illusion égalitaire », pour reprendre l'expression de Marie Duru-Bellat ? La fracture scolaire est-elle condamnée à augmenter en raison des inégalités sociales croissantes ? Cela dépend-il des réponses territoriales ou de réponses globales ? L'article de Pierre-Louis Gauthier apporte des éléments de réponse, en s'appuyant notamment sur des analyses de la Chine, l'Uruguay, l'Espagne, l'Allemagne et la France, en s'interrogeant sur les liens entre déséquilibres sociaux et inégalités scolaires.

Tout enseignement se réfère à des valeurs (explicitement ou implicitement) et décide des contenus ${ }^{5}$. L'École est de plus en plus souvent interpellée sur ce qu'elle enseigne ; mais qu'apprennent réellement les élèves $?^{6}$ La mondialisation a-t-elle un impact sur ce registre ? Florence Robine et Roger-François Gauthier proposent leurs réponses à ces questions, éclairées par ce qui se passe en Angleterre, en Suède, au Sénégal, en Inde et en France, en incluant le rôle du marché et d'Internet. Ils se demandent s'il existe des convergences dans ce monde bigarré.

La mondialisation favorise le développement des marchés qui, dialectiquement, poussent à renforcer la mondialisation. Pour autant, l'éducation est-elle devenue un simple segment du marché scolaire se demande Bernard Cornu ? S'appuyant sur les cas de la Russie, de la Corée, du Maroc, de la Finlande et sur les travaux de l'OCDE, il met en relief les articulations entre les registres techniques (où et comment peut-on apprendre ?) et politiques (que veulent les États ?). Il montre que se dessinent déjà, ici ou là, des systèmes hybrides.

\section{ESSAI DE PERSPECTIVE GLOBALE}

Michel Lussault (géographe, président de l'université de Lyon), avec finesse et humanisme, tire les premiers enseignements de ces points de vue en tension, mais complémentaires. Il aide à discerner la complexité du sujet et note, en particulier, l'importance des régulations politiques. Il s'interroge sur le rôle futur de l'École comme ascenseur social et sur l'impact de l'autonomie des unités scolaires. Son article, charnière de ce dossier, tient lieu de première synthèse soumise ensuite aux réactions d'experts internationaux. Chacun des propos tenus (plutôt philosophiques et institutionnels) éclaire, récuse, relativise, complète et remet en perspective ce qui précède. À leur façon, les intervenants affirment qu'un autre monde et qu'une autre École sont possibles.

5. Voir : Revue internationale d'éducation de Sèvres $n^{\circ}$ 50, avril 2009, «En classe : pratiques pédagogiques et valeurs culturelle », coord. Anne-Marie Bardi et Maroussia Raveaud.

6. Le numéro 43 de la Revue internationale d'éducation de Sèvres, "Que savent les élèves ? ", décembre 2006, est consacré à ce sujet. 
Roger Dale voit dans les évolutions actuelles un changement radical du contrat social et explique son point de vue ; Mamadou N'Doye (ancien ministre de l'Éducation, président du Réseau éducation pour tous en Afrique) apporte la vue de son continent et bien au-delà : il invite à repositionner l'humanité dans le développement ; David Istance (expert à l'OCDE), cite l'existence de corrélations entre satisfaction de l'école et proximité du rôle des parents d'élèves et explique pourquoi il faudrait s'éloigner du modèle quantitatif de la massification ; enfin, Tapio Säävalä (Commission européenne) observe que l'École change, mais plus lentement encore que l'Église; il formule ses réflexions en termes de formation tout au long de la vie, de compétences clés, de dialectique entre Internet et spécificités territoriales. Il rappelle que les écoles peuvent apprendre les unes des autres.

L'article de Riccardo Petrella développe un inhabituel parallélisme entre l'eau et l'éducation : deux biens communs particulièrement précieux et à préserver. Son approche écologique, en termes de développement durable, répond, à sa façon, à l'impulsion économique initiale de Thierry Breton. Il insiste sur les responsabilités des acteurs et des systèmes, en mettant en exergue de fortes valeurs éducatives.

Enfin, en tant que grand témoin du colloque et avec le recul nécessaire, Bernard Charlot (professeur émérite à l'Université Paris 8 et professeur visitant de l'Université fédérale de Sergipe, Brésil) replace l'ensemble de ce dossier dans une ultime perspective globale. Il observe des convergences, mais surtout une pluralité de modèles qui ne peuvent s'exporter directement d'un territoire à un autre, notamment pour des raisons culturelles. Rebouclant à sa façon sur le thème des communautés introduit par Thierry Breton, il s'affirme convaincu que l'on se dirige vers plus de différenciation interne aux systèmes scolaires et il explique pourquoi.

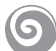

Il apparaît clairement que les modèles d'école des siècles derniers ont déjà dépassé leurs limites. Aucun ne fait d'ombre à un autre, mais nul ne peut s'imposer comme unique modèle général. Des pistes nouvelles sont ouvertes, toutefois elles peinent à se dessiner. S'il était besoin d'en administrer la preuve, la lecture de ce numéro montrerait à l'évidence, que l'école du XXI e siècle reste à inventer. Or, nous dit Michel Lussault, « il faut que l'École apprenne à composer avec la variété des mondes». Son avenir sera donc pluriel et hybride. 